\title{
THE COMPUTER AIDED EXERCISE - AN ALTERNATIVE OF THE CONVENTIONAL EXERCISES IN THE ARMED FORCES
}

\author{
Nikolay VRAIKOV and Alexi NAIDENOV
}

$\mathrm{D}$ esigning and building simulation systems is a dynamically developing area, in which, for relatively short time and under definite conditions, good results can be achieved. Our army has gained significant experience in the development and use of various simulation systems. The participation in a great number of seminars, presentations and computer aided exercises gives us the opportunity to analyze the achievements in the area of development and use of simulation systems in the education and training process of the staffs at various levels.

What we should start with when discussing the problem of computer aided exercises $(\mathrm{CAX})$ is to disintegrate the whole, i.e. the simulation, to define its components and based on that, to find the place of the CAX in it.

Thus the whole - the simulation-is a process of recreation and imitation of the reality (in complex systems) with a definite precision, using several interrelated and interdependent objective and/or abstract (mathematical) models, working as an integrated whole, subject to a common concept and plan. Therefore, the characteristic features of the simulation are the following:

- the simulation is not a fixed process, not a state already reached but a strictly defined process of imitating a specific object, its characteristics and way of functioning;

- existence of a number of mathematical models, different and yet compatible, complementing each other and working under a common definition and medium;

- $\quad$ each of these models solves a strictly specific problem, which is a part of the general re-creation of the reality. 
Talking about simulation, we must have in mind that according to their technical qualities and potential capabilities we can differentiate three basic simulation methods:

1. Live simulation;

2. Virtual simulation;

3. Constructive simulation.

Under live simulation we understand training or exercises with real troops and real combat equipment on the terrain and in conditions as close as possible to the real ones.

Under virtual simulation we understand instruction and training in an artificially created environment, where through simulators used for the actions of the operator or the crew, the behavior of the entire system is represented. A primary task here is to create as genuine as possible imitation of the original system and its behavior. Two basic levels can be differentiated with the virtual simulation:

- Level I - These are the so called individual training simulators. Here we do not mention the interacting computer audio-visual means of training and visualization, since they rather mark the transition from the purely applied use of computer technologies to the simulation. For us here, of a specific interest are the simulators. They are used for formation and maintenance of definite habits and skills of the individual soldier (commander). The trainers are basically related to the use of definite weapon systems and means. In other words, the realization of the aims and tasks at the first level is achieved through the so called simulators. Examples in this respect are: movement and flying simulators; shooting simulators; duel fighting simulators, etc.

- Level II - The so called higher class simulators used for tactical training of crews, commanders and staffs (Tactical training). They are used to confirm and develop the concepts of conducting combat actions from crew level to company level, in a realistic and challenging combat which is artificially created. The purpose of using them is to put together the tactical actions of crew, platoon and company.

The next simulation method is the constructive simulation. Under constructive simulation we understand the application of simulation models using instruments for analysis, which are capable of assessing and solving the consequences from the decisions made. Here, the acceptance of the system as a whole is always determined by the likelihood of the simulation results. In the constructive simulation, two levels can be distinguished, too: 
- Level I - Computer Aided Exercises (Command and Staff Training) - The most modern and effective method of training of commanders and staffs at a battalion level and above. They are aimed at a formation and keeping up definite habits with commanders and staffs related to control of subordinates and also at optimization of staff procedures and functions.

- Level II - Expert systems - Meant mainly for solving defense planning problems and strategic analyses. The simulation systems of that level support the decision making process in the area of military planning, military doctrine development and analysis of interoperability issues. They are also widely applied in the preparation of comparative analyses when making alternative decisions (different in number, structure and type of armed forces), interaction among the various subsystems (subcomponents) of the armed forces and operational strategies.

As basic requirements for the development of the simulation systems used in CAXs the following can be defined:

- the software should be interoperable with the equipment and information systems used for forces control;

- the simulation systems should be capable of configuring depending on the user's requirements, composition and level of the participants and in compliance with the exercise objectives;

- an interface, corresponding to the international standards should be used, which will allow for a common use of a number of simulation systems with the purpose of conducting comprehensive multinational CAXs;

- centralized coordination in the development and the use of simulation systems for CAXs;

- the simulation systems should be in a didactic, logic and technological uniformity.

- all simulation systems should use a common basic data-bases (for the area, vulnerability, weapon systems capacities, etc.)

Having thus defined, although in most general terms, the place of the CAX, we will further try to define its nature and outline its basic characteristics.

The Computer Aided Exercise is an interactive and non-traditional method of training, based on the application of a wide range of computer mathematical models, providing a true representation of the combat actions and offering opportunities for making certain deductions out of which a conclusion about the eventual subsequent behavior of the trainees in a real environment could be made. In other words, it can be looked upon as a super-modern method of training based on the use of modern information technologies and microprocessor equipment and the application of 
animation and simulation techniques on the background of an adequate visual and acoustic image.

Before moving on to the functional and technological structure of the CAX, let us focus on its advantages. In general terms they can be brought to the following:

1. It is a cheap and highly effective alternative of the conventional types of exercises, shortening considerably the time necessary for achieving the same results through conventional exercises. The interactive simulation systems used in the CAX staff exercises allow for a fast and quality planning, assessment, decision making, working out of the plan and tactics of actions in definite pre-set initial conditions. In that way, as a result of playing out a number of situations at a minimum outlay of material and human resources, the actions of various institutions, units and individuals working together to a common end are coordinated.

2. Environmental protection - The opportunity to play out various moves, simulated through mathematical models excludes the likelihood of polluting and damaging the natural environment due to discharging harmful substances from the machines. In that way, considerable funds needed for its recreation are saved.

3. Decreased risk situations - The opportunity for playing out to a degree of perfection various moves, simulated through computer models decreases the dangers when applying to practice what has been learned, since no wrong move can lead to injuries or eventual loss of a human life.

4. Saving of time and giving opportunities for multiple repetitions and play outs Judging from international experience the training of staffs and commanders at various levels through CAXs saves up to $60 \%$ of the time needed for acquiring the same knowledge and skills in practical training. Besides, through the simulation systems it is possible to play out repeatedly various scenarios of a certain leader's decision and also to react quickly and timely to emergency situations, i.e., to receive timely information about an expected turn in introducing the second echelon, air assault disembarkation into a certain area, etc. All that allows for a choice and working out of a more efficient training strategy.

5. Economy of resources - That feature has always been topical but in the current poor economical indices of our country, its significance is even greater. If we compare for example the funds, which will be needed for conducting an exercise with troops on the terrain to those needed when using the simulation system of CAXs, it will become clear that they are incomparable. Thus, for example, to conduct a brigade level CAX approximately DM 7000 will be needed, while an exercise achieving the same results but conducted through deployment of equipment on the terrain will already cost DM 240000. 
6. Optimization of the decision making process - The CAXs offer opportunities for studying the process and techniques of decision making. Based on the statistical information obtained, it is possible to enter repeated corrections to improve them.

7. Improvement of the communications and structures of the information flows, running among the main officials from the particular body of authority.

8. Raising of the quality of the exercise - The teams preparing and conducting CAXs are usually composed of experts with the best training. Furthermore, the simulation system is absolutely unbiased, so that classes of a high quality are offered in compliance with the preparedness level and specific physical and psychological state of the trainers and trainees.

9. Increased sense of one's own worth and motivation - The opportunity for the trainee to achieve independently and individually the particular objectives and follow the results of his or her decisions during the exercise leads to an increased sense of their worth and motivation unlike the conventional frontal communication.

10. Orientation to the future - As a result of the use of the most modern equipment and information technologies, the volume of knowledge of the capabilities of the simulation models and CAXs is strongly increased. The simulation models, with their constant updating and improvement allow for a complete application of the latest achievements in the area of the modern interactive methods of instruction, training and fitting together of the staffs. Furthermore, they make it possible to conduct CAXs from the staffs' permanent locations and from their wartime command posts, the so called distributed CAXs. Distributed CAXs are the next step and level of using this instrument in staff training.

Specifically in the Bulgarian army, the existing simulation system for CAXs was put into operation as early as 1996. It is intended for conducting Command Post CAXs at a brigade staff level. This system is open and allows for:

- perfection of the existing models, as well as inclusion of new ones aimed at achieving a higher degree of reliability in controlling the combat actions. It is compatible with the local information computer network of the staffs trained and in future it is expected to work in a regional regime;

- all trainees from the leadership bodies are to fulfil in full their functional duties on decision making, planning the operation (battle), organizing the combat actions, ensuring a firm and continuous control, maintaining the interaction and organization of the overall support in compliance with the generally accepted techniques;

- carrying out staff training and uni-/multistage exercises of the tactical and operational staffs, without or with participation of troops; 
- many-sidedness of the exercise when decision making and control of the forces takes place in the conditions of a modeled and close to reality clash with the enemy;

- assessment of the terrain and organization of the interaction of the staffs trained using a geographical information system for terrain analysis with a specific purpose;

- accumulation of certain knowledge and skills for situation assessment, decision making and forces control;

- collection, storage, processing, display and dissemination of information about the situation with all its elements;

- collection of information necessary for exercise analysis;

- training of the staffs at training centers specifically established for that purpose and, in future, also at permanently located posts;

- $\quad$ storing the information and the versions of the decisions made and played out.

As we already mentioned, the CAXs are a form of staff training, in the course of which simulation systems are used, providing a true presentation of combat actions. They are developed as interactive computer-based games and are intended for training of officers and staffs for fulfillment of their wartime functions.

Depending on the training aims set, tasks to be solved, trainees composition, degree of detail in forces representation, weapon systems, terrain and impact of other factors, the CAXs are subdivided into three types: group, detailed and general.

The group exercises have a limited number of participants and hierarchy levels. With them, a small number of problems are worked out which reflect one episode of the combat actions using general simulation model. The modeling is fast and allows for focusing on the exercise objectives.

The general exercises have wider objectives and participating in them are officials, whose functional duties are related to the achievement of the objectives. With those exercises the individual episodes of the combat actions are not simulated in detail but they are based on more general principal positions. The functioning of the staff trained is in real time which can also be made shorter.

Detailed exercises are those exercises with which complex objectives are set. Participating in them are a large number of organizational entities and in their modeling lower organizational levels and a larger number of factors affecting the combat actions are taken into account. With them, a system of models of the Services, 
Branches and special forces, and the types of support, is used. The exercise runs in real time.

Each CAX is characterized by definite technological and functional structures.

The technological structure is intended for data collection, display, dissemination and processing, and for formation and presentation of the modeling results. It ensures the dynamics of the exercise concept and makes the situation look real. Its main components are the simulation system of combat actions modeling, the computers themselves, the technical equipment and the communications infrastructure.

The simulation system for combat actions modeling allows for simulation of various aspects and functions of the combat actions, receives the input data for the modeling, guides the events from the operations (battle) dynamics and figures out its results.

It consists of the following systems:

- a model of the all-arms operation (battle). It provides a simulation of the combat actions of the battalions, brigades and corpses, making a complex accounting of their resources. It allows for modeling of the effect from using conventional and other types of ground-based weapons. It allows for playing out all characteristic actions of the Land Forces on the background of definite geographical system, modeling completely the terrain relief and the operation of a large number of different types of weapons;

- a model of the Air Force - It simulates the aspects of the combat actions with conventional forces and equipment of the aviation and the air defence. This is a threedimensional and spatial model of the actions during daytime and nighttime, in simple and complicated meteorological conditions. Participating in the model are the airbases, the missile complexes of the air defence of all classes and types, and the components of the radar system. It gives a detailed account of the armaments and resources of ammunitions and POL;

- a model of the Navy - It simulates the spectrum of actions and factors of maritime battles, including convoying and transfer of forces;

- model of reconnaissance and electronic warfare - It simulates the process of reconnaissance of the enemy's sites with the full complex of bodies and techniques of actions, electronic jamming of the radar and communications systems, counteraction against the enemy's reconnaissance equipment and the radio-electronic protection of the friendly equipment for control provision;

- model of the operational and combat support and model of the logistic support It simulates the systems for operational, combat and logistic support; 
- model of NBC - It simulates a creation of a complicated radiation and chemical situation;

- model of the control system - It simulates the command, control and communications (C3) system.

Within the models the users work through a common users interface. Through it, the initial data are entered and the results from the entire modeling process are received.

The technical equipment for conducting CPX CAXs is usually set up into specialized training centers.

The computers, on which the models work and on which the communications system is based, are various types and with various capacities. There are two basic types of computers:

- workstations;

- servers.

The workstations can be:

- processor - not lower than 1486, Pentium;

- clock frequency - not less than $300 \mathrm{MHz}$;

- RAM - not less than $32 \mathrm{MB}$;

- hard disk volume - not less than $5 \mathrm{~GB}$;

- monitor - SVGA;

Servers:

- processor - RISC;

- operational memory - expandable up to $640 \mathrm{MB}$;

- disk space - built in 12-16 GB, maximum - $200 \mathrm{~GB}$;

- output - not less than 100 SPEC int 92;

For the local computer network:

- IEEE 802.3/Ethernet;

- 10 BASE - T;

The local computer network itself includes three types of workstations:

- for work with verbal and table battle documents (operational directives, battle orders, battle instructions, battle reports, information reports, briefings, etc.);

- for work with operational information, using topographic basis;

- for linking with the models for combat actions.

These workstations are the minimum necessary for conducting CAXs. 


\section{For UPS:}

- for server - not less than $1200 \mathrm{Bt}$;

- for workstations - not less than $600 \mathrm{Bt}$

The simulation system which is used for conducting CAXs works with the following system software:

- for the work stations - Windows 95;

- for the server - UNIX OC for RISC, Windows NT 4.0;

- for the local computer network - Windows NT 4.0, TCP/IP;

- ORACLE database management system.

The applications for data input and output in the database are built with the following computer languages:

- Technological development medium DELFI 2;

- Technological development medium Borland C++4.0, 5.0]

- Technological products from ORACLE database management system.

The communications in the CAXs insures the distribution of the processed information in the preparation and course of the exercise. It is the communications and the computer interface that provide the conduct of distributed CAXs, which means to use the simulation models from remote locations, where the exercise participants are (the places of permanent location, wartime command posts, special training centers, etc.) and exchange of video and verbal information.

Figure 1 presents organizational structure and information flows in a CAX. The functional structure of a computer aided exercise is represented on figure 2 .

As an organization the participants in the preparation and conduct of CPXs are divided into a directing staff, a trained staff, a group maintaining the modeling system and a supporting group.

The directing body is responsible for the preparation and conduct of the exercise and achievement of the exercise objectives. It can have the following structure: director, chief of staff and directing staff.

The director of the exercise is responsible for the timely preparation and conduct of the exercise at a high organizational and methodological level. He is determining the theme, the training objectives and problems, the area of the exercise and the time limits for the preparation of the documents.

The chiefs of staff of the directing body is to organize the staff's activity and control the development of the documents necessary for the exercise conduct, organize the work of the staff during the exercise. 


\section{Organizational Structure and Information Flows} of a Computer Assisted Exercise

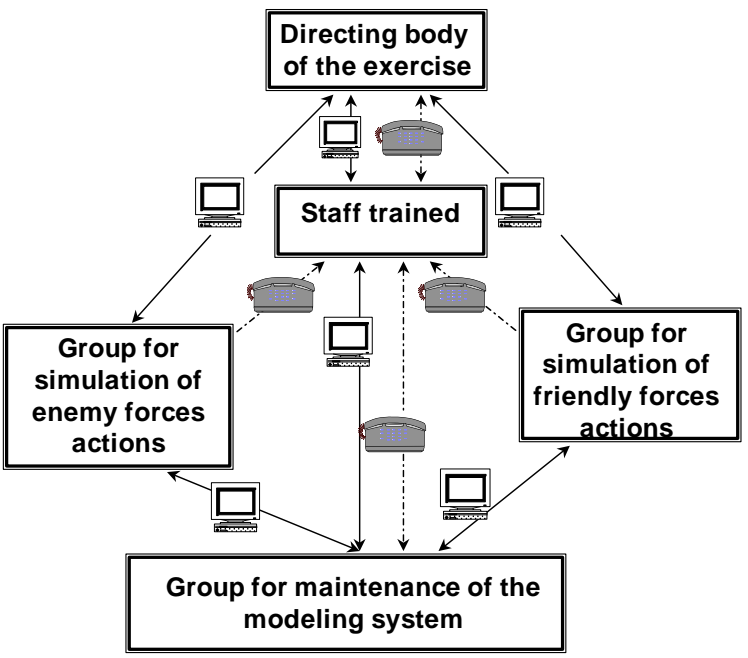

Connection with the simulation system

- Telephone connection

Figure 1. Organizational structure and information flows in a CAX.

\section{Functional Structure of a Computer Assisted Exercise}

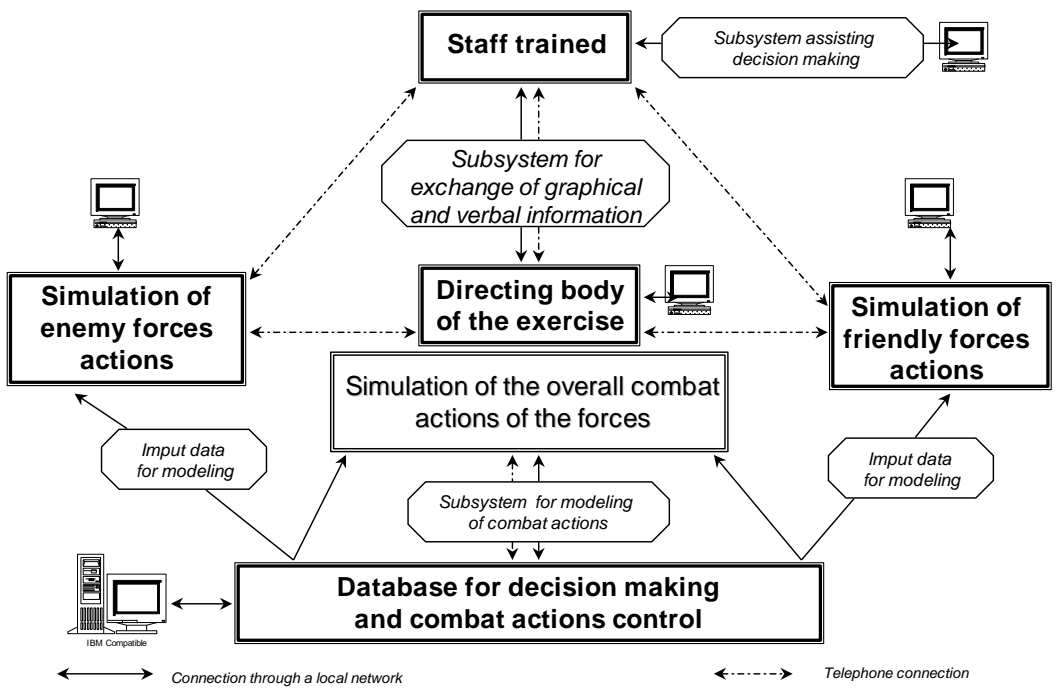

Figure 2. Functional structure of a CAX 
The directing staff is a basic body in the preparation and conduct of the exercise. It is to develop all documents related to the exercise. It includes a group for interaction, a group for simulation of the friendly forces actions, a group for simulation of the enemy forces actions and a group for control and analysis.

The group for interaction simulates the actions of the superior staff versus the staff trained and the staffs of its neighbors or realize the functions, which are not worked out during the exercise.

- The group for simulation of the friendly forces actions simulates the actions of the subordinates to the staff trained, staffs or forces, depending on whether the exercise is a two-stage or a multi-stage one.

- The group for simulation of the enemy forces actions develops the concept of the counteracting party, models the actions on the ground, water and air in the course of the operation (battle), thus counteracting the decisions made by the trainees.

- The group for control and analysis controls the course of the exercise, ensures the achievement of the objectives and analyzes the activity of the staff trained.

The staff trained is in its real personnel composition, all officials performing their functional duties.

The maintenance group prepares the model, observes the modeling process, takes care of the stable work of the overall system, keeps track of the network and communications equipment and maintains the operating data exchange system.

The supporting group provides for the life support of the exercise participants.

The organization itself and the conduct of the exercise determine the run of several basic information flows.

The first one includes the whole information generated and exchanged through the simulation model. Included in this information cycle are: the exercise directing staff, the group simulating the friendly forces actions, the group simulating the enemy actions and the modeling group. The information running here is graphical and verbal. Through it the clash of the two warring parties and the course of the combat actions. This information cycle insures a reliable control and, when necessary, interference in the course of the exercise by the modeling group. The medium for this information flow is the network built, which connects the servers, the work stations and the equipment for the output of graphical and verbal information.

The second basic information flow is the flow running between the directing staff, the training staff and its subordinate units. The realization of the information exchange is 
accomplished through the communication channels established by means of the available communications means - telephones, radio stations, etc. It provides for the exchange of current information above all between the staff being trained and their subordinates in connection with the changed situation. The reliable functioning of this information flow determines the quality of the exercise conduct. All changes of the situation which are displayed at the work stations using the available communications equipment are reported by the subordinates to the commander of the staff trained, since he is unable to follow the change of the situation through a workstation. He receives information about the course and development of the combat actions from his subordinate commanders' reports only, as it is in a real situation. The functioning of this information flow provides for reporting of the situation by the commander of the staff trained to the directing staff, for assigning the tasks to the subordinates and receiving directions from the directing staff.

The third basic information flow during the conduct of the CAX insures the exchange of graphical and verbal documents between the exercise directing staff and the staff trained. This information channel is used for sending battle orders, reports, instructions and visual graphical information. It is realized through the local network and computers with special designation.

In conclusion, we must emphasize that the solutions of the issues related to the use of simulation systems in the training process of the Bulgarian Armed Forces in the next several years will be one of the main directions in the army's activities. A basis for that is the Military Doctrine of the Republic of Bulgaria and the Plan for Organizational Development of the Armed Forces till 2004.

The subdivision of the Armed Forces according to their designation into Rapid Reaction Forces, Defence Troops, Territorial Defence Troops and Reserve Forces, and the new functions of the Armed Forces in accordance with the Military Doctrine of the Republic of Bulgaria as deterring and defensive functions, peace-support functions, humanitarian and rescue functions, international and social functions necessitate intensification of staff preparation, implementation of new forms and methods of training. This has been reflected in Article 95 of the Military Doctrine "In the forces and staff training, alongside the conventional methods, simulator and computer supported forms of training are also used..."

Theoretically, the issue of using simulation systems in the Bulgarian Armed Forces is further developed in Plan 2004, where it is said that "the methods of staff training will be perfected for acceptance of the modular principle of preparation and conduct of simulation exercises and exercises conducted according to the standards and procedures established in the armed forces of the NATO member states and in order to achieve the interoperability objectives and adapt the standardization documents 
and staff procedures to our conditions, the relative share of the Computer Aided Exercises will be increased..."

All that determines as a primary task to be realized by the army during the next coming years the mastering of new and more effective forms of training of the staffs and forces based on effective use of the simulation systems.

\section{References:}

1. Arfeev, N., "Using Computer Technologies in Troops Training," Military Thought (Voennaya myisl) 12 (1988). - in Russian.

2. Alexi Naidenov, Nikolay Vraikov, "The Computer Supported Exercise - A Modern Way of Knowledge Acquisition," Military Journal 105, 1 (1998), 48-52. - in Bulgarian.

3. Valentine Penev, "Simulation Modeling in Military Affairs: Status and Perspectives," Information \& Security 1, 1 (Summer 1998), 91-102.

4. Modeling and Analysis of Conventional Defense in Europe, ed. R. Huber (Plenum Press, 1996).

5. Huber, R., L. Jones, and R. Jones, Military strategy and tactics computer modeling of land war problems (Plenum Press, 1985).

6. User's Manual for the Warrior Preparation Center Ground War Simulation Model. WPC (March 1991).

NIKOLAY VRAIKOV, Ph.D., graduated from "V. Levski" Army Academy in 1985. For several years he has served at a number of garrisons of the Bulgarian Army, after which in 1991 he received doctoral degree in mathematical processing of empirical data. In the period of 1991-1994 he worked as a research associate at "G.S. Rakovski" War College. In 1996 he completed the full course of education of "G.S. Rakovski" War College in the specialty of Automation in Management. Then for one year he worked as a senior assistant at "Automation Department" of the General Staff where he was in charge of the computer systems used by the Navy and the conduct of computer assisted exercises. Since 1996 he is working as a senior assistant at "Exercises, Training and War Games" Department at the Operations Directorate of the General Staff on the issues of simulation systems for CAX, as well as on problems of collection and dissemination of their results, inference and lessons learned.

ALEXI NAIDENOV is an officer, chief of "Exercises, Training and War games" Department at the Operations Directorate of the General Staff. In 1977 he graduated from "V. Levski" Army Academy. Then he served in a number of Land Forces units. In 1986 he graduated from "Frunze" War College in Russia. After that he consecutively took positions at various staffs Corps HQ, Land Forces Headquarters and General Staff of the Bulgarian Armed Forces. Since 1993 he is involved in designing and implementing the policy of using simulation systems in staff and force training. 